\title{
Systemic therapy options following first-line chemoimmunotherapy in small-cell lung cancer
}

\author{
Jose M. Pacheco \\ Division of Medical Oncology, Department of Internal Medicine, University of Colorado Cancer Center, Aurora Colorado, USA \\ Correspondence to: Jose M. Pacheco MD. Assistant Professor, Thoracic Oncology and Developmental Therapeutics, 1665 Aurora Court, Room 5309, \\ Mail Stop F704, Aurora Colorado 80045, USA. Email: jose.m.pacheco@cuanschutz.edu.
}

\begin{abstract}
Nearly all patients with extensive-stage small-cell lung cancer (ES-SCLC) relapse following firstline etoposide plus platinum (EP) with or without immune checkpoint inhibition. Topotecan and amrubicin are chemotherapies approved for these patients. The toxicities of these chemotherapies are significant and survival when treated with these regimens is minimal. The programmed death-1 (PD-1) inhibitors nivolumab and pembrolizumab are unlikely to be effective for patients who develop progressive disease on first-line chemoimmunotherapy. Newer systemic therapies (e.g., lurbinectedin and temozolomide plus polyADP ribose polymerase inhibition) have demonstrated greater response rates than topotecan, amrubicin or PD-1 inhibitors. The data on these newer systemic therapies and other agents that may soon enter clinic are reviewed in this manuscript. Additionally, some of the key questions arising following clinical trials of these newer agents are highlighted.
\end{abstract}

Keywords: Small-cell lung cancer (SCLC); lurbinectedin; temozolomide; second line; subsequent line

Submitted Feb 25, 2020. Accepted for publication Mar 26, 2020.

doi: $10.21037 /$ jtd.2020.03.67

View this article at: http://dx.doi.org/10.21037/jtd.2020.03.67

\section{Introduction}

The standard of care first-line systemic therapy for extensive-stage small-cell lung cancer (ES-SCLC) is now a programmed death ligand-1 (PD-L1) inhibitor plus etoposide plus platinum (EP). There is no standard next line systemic therapy following development of progressive disease on this chemoimmunotherapy regimen. Topotecan and amrubicin, as well as the programmed death-1 (PD-1) inhibitors nivolumab or pembrolizumab have been approved as subsequent line therapies in different parts of the world. Nivolumab or pembrolizumab monotherapy are unlikely to be effective following development of progressive disease on first-line therapy with a PD-L1 inhibitor plus EP. Topotecan and amrubicin have limited benefit in this setting and significant toxicity (1-4). Recent clinical trials of lurbinectedin or the combination of temozolomide plus poly ADP ribose polymerase (PARP) inhibitors have shown encouraging response rates in patients with ES-SCLC treated in the second or subsequent line setting (5-8). In this review we summarize the data on these and other systemic therapy options for patients who develop progressive disease on first-line chemoimmunotherapy.

\section{Methods}

Trials were identified by searching PubMed without date limits, abstracts from major medical society meetings since 2015 (American Association for Cancer Research Annual Meeting, American Society of Clinical Oncology Annual Meeting, European Society of Medical Oncology Annual Meeting, International Association for the Study of Lung Cancer World Conference on Lung Cancer), presentations from the International Association for the Study of Lung Cancer Targeted Therapies of Lung Cancer Meeting in 2020, and clinicaltrials.gov using the keywords: lurbinectedin, lurbinectedin plus doxorubicin, PM01183, temozolomide, temozolomide plus veliparib, temozolomide plus olaparib, talazoparib and small-cell lung cancer, topotecan, amrubicin, CAV and anlotinib. Trials 
not reporting efficacy data on small-cell lung cancer were excluded.

\section{Lurbinectedin}

Lurbinectedin impairs the formation of ribonucleic acid (RNA) by limiting the binding of transcription factors to their promotors $(5,6)$. This action may have antitumor activity in certain malignancies. Additionally, lurbinectedin decreases transcription within tumor-associated macrophages, which may lead to decreased tumor cell proliferation, decreased tumor cell survival, improved antitumor immunity and decreased angiogenesis $(5,6)$.

Lurbinectedin is not approved for treatment of ESSCLC. However, multiple clinical trials have evaluated lurbinectedin as either a single agent or in combination for second or subsequent line therapy of ES-SCLC $(5,6,9)$. A new drug application has been submitted to the Food and Drug Administration (FDA) for lurbinectedin monotherapy in relapsed SCLC. A decision on this approval is expected by August 2020 (10). Additionally, an expanded access program for single agent lurbinectedin has recently opened. The data supporting the FDA new drug application are summarized below, as are trials that have evaluated lurbinectedin in combination with other chemotherapies.

\section{Lurbinectedin monotherapy}

A phase II study evaluated lurbinectedin monotherapy for ES-SCLC patients who had received $\geq 1$ prior line of chemotherapy. There were 105 patients treated. Only 4 patients had a history of baseline central nervous system (CNS) metastases. The CNS metastases in each of these 4 patients were asymptomatic and previously treated. Patients with Eastern Cooperative Oncology Group (ECOG) performance status of $0-1$ accounted for $92.4 \%$ of the study population. Only $7.6 \%$ of treated patients had received prior immunotherapy. Lurbinectedin was dosed intravenously at $3.2 \mathrm{mg} / \mathrm{m}^{2}$ every 3 weeks until development of progressive disease or other discontinuation criteria were met. The median follow-up was 17.1 months $(6,11)$.

The investigator assessed confirmed objective response rate (ORR) was $35.2 \%$. Platinum sensitive patients had a higher ORR at $45.0 \%$ (27 of 60 ) when compared to platinum resistant patients who had an ORR of $22.2 \%$ (10 of 45). Similarly, the median investigator assessed progressionfree survival (PFS) was 3.9 months. The PFS was greater in platinum sensitive patients when compared to platinum resistant patients, median PFS of 4.6 versus 2.6 months. Median overall survival (OS) was 9.3 months. The OS was also greater in platinum sensitive patients when compared to platinum resistant patients, median OS of 11.9 versus 5.0 months, 1 -year OS of $48.3 \%$ versus $15.9 \%$. This drug was relatively well tolerated, with the most common adverse events consisting of cytopenias (Table 1) $(6,11)$. Dose delays and dose reductions each occurred in approximately $25 \%$ of patients. About $20 \%$ of patients required administration of granulocyte colony stimulating factor (G-CSF) $(6,11)$.

\section{Lurbinectedin plus doxorubicin}

A phase $\mathrm{I} / \mathrm{Ib}$ trial evaluated this combination in patients with ES-SCLC. The recommended phase II dose (RP2D) was doxorubicin $40 \mathrm{mg} / \mathrm{m}^{2}$ on day 1 plus lurbinectedin $2 \mathrm{mg} / \mathrm{m}^{2}$ on day 1 , with both medications being administered every 21 days. Doxorubicin was administered until a total dose of $450 \mathrm{mg} / \mathrm{m}^{2}$, development of progressive disease or other discontinuation criteria were met. Lurbinectedin monotherapy was continued at a dose of $4 \mathrm{mg} / \mathrm{m}^{2}$ in patients who had stable disease or better following completion of combination therapy. Lurbinectedin was administered until development of progressive disease or other discontinuation criteria were met. There were 27 patients treated at the RP2D, only 1 of these patients had known CNS involvement. The number of patients having received prior immune checkpoint inhibition was not available (5).

The ORR at the RP2D was $37 \%$. The median PFS was 3.4 months and the median OS was 7.9 months. This regimen had a high amount of grade $\geq 3$ adverse events, mainly consisting of cytopenias. Neutropenia was the most common grade $\geq 3$ adverse event, with an incidence of $93 \%$ (Table 1) (5). It is important to note that primary prophylaxis with G-CSF was not administered with this regimen and perhaps should have been. The investigators on this trial state that the neutropenia was well managed with G-CSF and dose reductions (5).

Patients in this trial with a chemotherapy free interval (CTFI) $<30$ days were observed to have very poor outcomes with this chemotherapy combination. Thus, an exploratory analysis was done excluding this poor prognosis group. When doing this the ORR was $48 \%$ (10 of 21), median PFS was 5.3 months and median OS was 10.2 months. Efficacy outcomes were much better for platinum sensitive patients 
Table 1 Emerging second or subsequent line systemic therapy options for extensive-stage small-cell lung cancer

\begin{tabular}{|c|c|c|c|c|c|c|}
\hline Therapy & $\begin{array}{l}\text { Patient } \\
\text { number }\end{array}$ & $\begin{array}{l}\text { Median lines } \\
\text { prior therapy }\end{array}$ & ORR & $\begin{array}{c}\text { PFS } \\
\text { (months) }\end{array}$ & OS (months) & Adverse events \\
\hline Lurbinectedin & 105 & 1 (range, 1-2) & $\begin{array}{l}35.2 \%(95 \% \\
\mathrm{Cl}, 26.2-45.2)\end{array}$ & $\begin{array}{c}3.9(95 \% \mathrm{Cl}, \\
2.6-4.6)\end{array}$ & $\begin{array}{c}9.3(95 \% \mathrm{Cl}, \\
6.3-11.8)\end{array}$ & $\begin{array}{l}\text { Grade 3-4 neutropenia } 22.9 \% \\
\text { Grade 3-4 anemia } 6.7 \% \\
\text { Grade 3-4 thrombocytopenia } 4.8 \% \\
\text { Grade 3-4 febrile neutropenia } 4.8 \%\end{array}$ \\
\hline $\begin{array}{l}\text { Lurbinectedin plus } \\
\text { paclitaxel }\end{array}$ & 7 & 1 (range, 1-4) & $71 \%$ & 4.8 & NA & $\begin{array}{l}\text { Grade 3-4 neutropenia } 85.7 \% \\
\text { Grade 3-4 anemia } 28.6 \% \\
\text { Grade 3-4 febrile neutropenia } 14.3 \%\end{array}$ \\
\hline Temozolomide & 64 & 1 (range, 1-2) & $\begin{array}{c}20 \%(95 \% \mathrm{Cl}, \\
11-32)\end{array}$ & $\begin{array}{c}1.6(95 \% \mathrm{Cl}, \\
0.9-3.0)\end{array}$ & $\begin{array}{c}5.8(95 \% \mathrm{Cl}, \\
4.2-7.0)\end{array}$ & $\begin{array}{l}\text { Grade 3-4 lymphopenia 30\% } \\
\text { Grade 3-4 thrombocytopenia 10\% } \\
\text { Grade 3-4 neutropenia 5\% } \\
\text { Grade 3-4 febrile neutropenia 2\% }\end{array}$ \\
\hline $\begin{array}{l}\text { Temozolomide plus } \\
\text { veliparib }\end{array}$ & 55 & 1 (range, NA) & $\begin{array}{c}39 \%(95 \% \mathrm{Cl}, \\
25-54)\end{array}$ & $\begin{array}{c}3.8(95 \% \mathrm{Cl}, \\
3.0-4.1)\end{array}$ & $\begin{array}{c}8.2(95 \% \mathrm{Cl}, \\
6.4-12.2)\end{array}$ & $\begin{array}{l}\text { Grade 3-4 thrombocytopenia 50\% } \\
\text { Grade 3-4 neutropenia 31\% } \\
\text { Grade 3-4 leukopenia } 24 \% \\
\text { Grade 3-4 lymphopenia 20\% } \\
\text { Grade 3-4 anemia 11\% } \\
\text { Grade 3-4 febrile neutropenia 4\% }\end{array}$ \\
\hline
\end{tabular}

Table 1 (continued) 
Table 1 (continued)

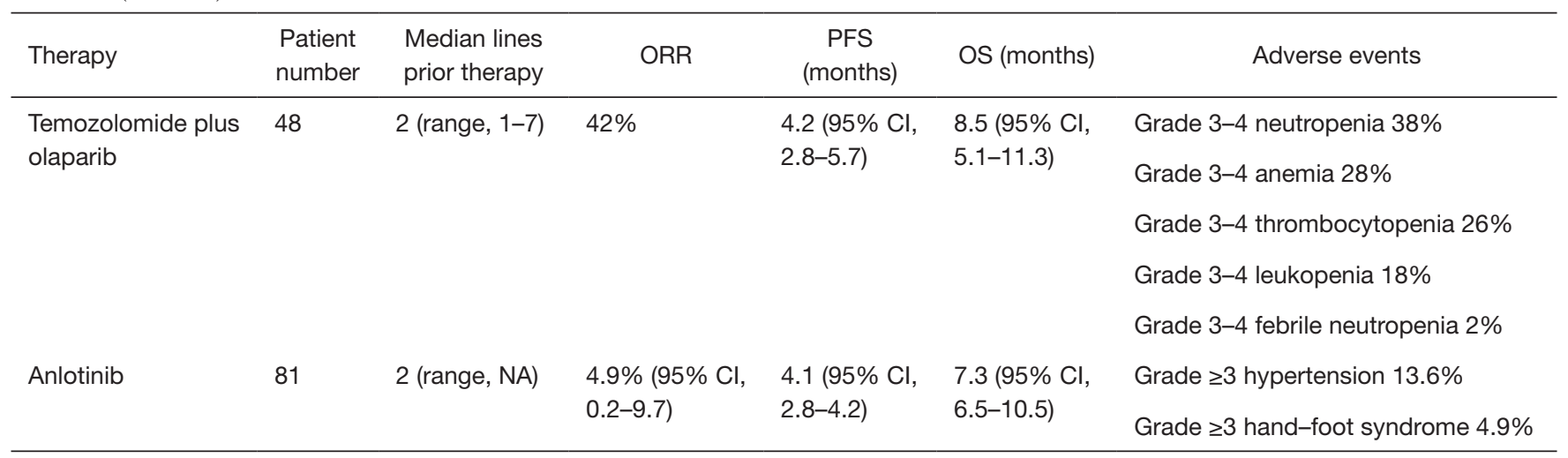

ORR, objective response rate; PFS, progression-free survival; OS, overall survival; $\mathrm{CI}$, confidence interval; NA, not available.

(CTFI $\geq 90$ days) when compared to platinum resistant patients. In platinum sensitive patients the ORR was $50 \%$ (9 of 18) and in platinum resistant patients the ORR was $11 \%$ (1 of 9). The median PFS was 5.7 months in platinum sensitive patients and 1.5 months in platinum resistant patients. Similarly, the median OS was 11.5 months in platinum sensitive patients versus 4.9 months in platinum resistant patients (5).

The high response rates seen in this trial were encouraging and prompted a randomized phase III trial called ATLANTIS. The ATLANTIS study excluded patients with a CTFI of $<30$ days because of poor outcomes seen in prior lurbinectedin trials. Patients must have received first-line platinum-based chemotherapy in order to be eligible, but they could not have received other chemotherapies. Patients with a history of CNS metastases were allowed, but these metastases must have been stable and patients could not have been taking steroids for management of CNS disease. There were 613 patients enrolled. They were randomized 1:1 between doxorubicin $40 \mathrm{mg} / \mathrm{m}^{2}$ on day 1 plus lurbinectedin $2 \mathrm{mg} / \mathrm{m}^{2}$ on day 1 of each 21-day cycle or standard chemotherapy (topotecan or cyclophosphamide plus adriamycin plus vincristine). The randomization was stratified based on ECOG performance status, CTFI ( $\geq 180$ versus $90-179$ versus $<90$ days), CNS involvement, prior PD-1 axis inhibition and investigator preference. This study required primary prophylaxis with G-CSF because of high rates of neutropenia seen on the phase Ib trial with doxorubicin plus lurbinectedin $(10,12)$. The final read out of this study is expected to occur during the third quarter of 2020 (10).

\section{Lurbinectedin with other systemic therapies}

Two phase I dose escalation studies evaluated lurbinectedin in combination with either irinotecan or paclitaxel in patients with previously treated ES-SCLC. There were $18 \%$ of patients treated with the irinotecan combination who received prior immunotherapy and $0 \%$ of patients treated with the paclitaxel combination that received prior immunotherapy. The RP2D of lurbinectedin in combination with irinotecan was irinotecan $75 \mathrm{mg} / \mathrm{m}^{2}$ on days 1 and 8 plus lurbinectedin $2.0 \mathrm{mg} / \mathrm{m}^{2}$ on day 1 every 21 days plus G-CSF as primary prophylaxis. The RP2D in combination with paclitaxel was paclitaxel $80 \mathrm{mg} / \mathrm{m}^{2}$ on days 1 and 8 plus lurbinectedin $2.2 \mathrm{mg} / \mathrm{m}^{2}$ on day 1 every 21 days. At all dose levels evaluated the ORR was $35 \%$ (6 of 17) for lurbinectedin plus irinotecan and $71 \%$ (5 of 7) for lurbinectedin plus paclitaxel (Table 1) (9). At the RP2D the ORR was $62.5 \%$ (5 of 8 ) for the combination with irinotecan and $80 \%$ (4 of 5) for the combination with paclitaxel. The median PFS was 5.4 months at all dose levels for the combination with irinotecan and 4.8 months at all dose levels for the combination with paclitaxel. Cytopenias are the predominant toxicities seen with these combinations (Table 1) (9).

\section{Future directions with lurbinectedin}

Trials are being planned to evaluate lurbinectedin in combination with immune checkpoint inhibitors. Additionally, there is thought being given to evaluating this drug in the first-setting. Ongoing trials of lurbinectedin are indicated in Table 2 (12). 
Table 2 Ongoing trials evaluating lurbinectedin in small-cell lung cancer

\begin{tabular}{lcccc}
\hline Trial & Phase & NCT number & Number of patients & Estimated completion date \\
\hline $\begin{array}{l}\text { Lurbinectedin with } \\
\text { irinotecan in solid tumors }\end{array}$ & Phase I/II & 02611024 & 150 & November 2021 \\
$\begin{array}{l}\text { Lurbinectedin with } \\
\text { olaparib in solid tumors }\end{array}$ & Phase Ib/II & 02684318 & 100 & October 2019 \\
$\begin{array}{l}\text { Lurbinectedin plus } \\
\text { atezolizumab small-cell }\end{array}$ & Phase I/II & 04253145 & 25 & February 2022 \\
$\begin{array}{l}\text { lung cancer } \\
\begin{array}{l}\text { Lurbinectedin versus } \\
\text { chemotherapy } \\
\text { (ATLANTIS) }\end{array}\end{array}$ & Phase III (randomized) & 02566993 & 613 & February 2020 \\
\hline
\end{tabular}

NCT, national clinical trials number.

\section{Temozolomide with or without a PARP inhibitor}

Temozolomide is an alkylating agent of deoxyribonucleic acid (DNA). Temozolomide has good CNS penetration and has demonstrated efficacy in primary brain tumors $(13,14)$. It has been evaluated in SCLC both as a monotherapy and in combination with PARP inhibitors $(7,8,15,16)$.

\section{Temozolomide or PARP inhibition as monotherapy}

Temozolomide monotherapy has been evaluated in relapsed ES-SCLC at two separate doses, $75 \mathrm{mg} / \mathrm{m}^{2}$ on days $1-21$ of a 28 -day cycle and $150-200 \mathrm{mg} / \mathrm{m}^{2}$ on days $1-5$ of a 28 -day cycle. In these trials the ORR ranged from $12 \%$ to $20 \%$. The median time to progression in these trials was 1.6 to 1.8 months. The median OS was 5.8 months. The main toxicities observed with monotherapy were cytopenias (Table 1) $(15,16)$. With temozolomide monotherapy responses have been seen in evaluable brain metastases which have not been previously irradiated, with an ORR of $0 \%$ ( 0 of 8 ) at $200 \mathrm{mg} / \mathrm{m}^{2}$ days $1-5$ and $38 \%$ (4 of 13 ) at $75 \mathrm{mg} / \mathrm{m}^{2}$ on days $1-21(15,16)$. As monotherapy the PARP inhibitor talazoparib did not demonstrate good efficacy in relapsed SCLC with an ORR of $9 \%$ in 23 evaluable patients (17). Preclinical models suggest enhanced efficacy from combining temozolomide and PARP inhibition (18-20).

\section{Combination therapy with temozolomide plus PARP inbibition}

A randomized phase II trial compared temozolomide plus the PARP inhibitor veliparib versus temozolomide monotherapy. Temozolomide was dosed at $150-200 \mathrm{mg} / \mathrm{m}^{2}$ on days $1-5$ of each 28-day cycle and veliparib or placebo were dosed at $40 \mathrm{mg}$ twice daily on days 1-7 (8). Additionally, a single arm phase $\mathrm{I} / \mathrm{Ib}$ trial evaluated temozolomide plus the PARP inhibitor olaparib. In this latter trial multiple dosing combinations were evaluated and the RP2D was determined to be temozolomide $75 \mathrm{mg} / \mathrm{m}^{2}$ daily on days $1-7$ and olaparib $200 \mathrm{mg}$ twice daily on days 1-7 of each 21-day cycle (7). The percentage of patients having received prior immune checkpoint inhibition on these temozolomide plus PARP inhibitor combination trials was not available $(7,8)$. The ORR with combination temozolomide plus PARP inhibitor appears to be greater than with temozolomide monotherapy. The ORR for temozolomide plus veliparib was $39 \%$ versus $14 \%$ with temozolomide alone (8). Combination temozolomide plus olaparib demonstrated a confirmed ORR of $42 \%$ (7). The clinical trial data do not demonstrate a clearly superior ORR for combination temozolomide plus PARP inhibitor in platinum sensitive versus resistant patients $(7,8)$. However, patient derived xenografts suggested the combination of temozolomide plus olaparib may be more efficacious in platinum sensitive tumors (7). With temozolomide plus veliparib, the ORR was $41 \%$ in platinum sensitive patients and $37 \%$ in platinum resistant patients. With temozolomide plus olaparib, the confirmed ORR was $47 \%$ in platinum sensitive patients versus $29 \%$ in platinum resistant patients $(7,8)$.

The numerically higher difference in ORR between platinum sensitive and resistant patients on the temozolomide plus olaparib study may have been in part due to the differences in classification of platinum sensitivity between the two studies. On the temozolomide plus veliparib study, platinum sensitivity was defined as 
relapse $\geq 60$ days following completion of platinum-based chemotherapy. On this study platinum resistance was defined as relapse during or within 60 days of completion of platinum-based chemotherapy or receipt of third-line therapy. On the temozolomide plus olaparib study, platinum sensitivity was defined as relapse $\geq 90$ days following completion of platinum-based chemotherapy $(7,8)$.

Survival outcomes were not clearly superior for combination temozolomide plus PARP inhibitor when compared to temozolomide monotherapy. The PFS with temozolomide plus veliparib was not statistically significantly improved when compared to temozolomide monotherapy, median PFS of 3.8 versus 2.0 months, $\mathrm{P}=0.39$. Similarly, the median OS was 8.2 months with combination therapy versus 7.0 months with temozolomide monotherapy, with 1 -year OS being $35 \%$ versus $30 \%$ respectively, $\mathrm{P}=0.50$ (8). With temozolomide plus olaparib the survival numbers appeared similar to combination temozolomide plus veliparib. At a median follow-up of 7.1 months for temozolomide plus olaparib, the median PFS was 4.2 months and median OS was 8.5 months (7). The most significant toxicities with combination temozolomide plus PARP inhibition are cytopenias and many patients treated with these combinations require dose reductions or dose interruptions $(7,8)$. However, unlike for lurbinectedin there is theoretical penetration of this combination therapy into the CNS and a potential biomarker that could predict for efficacy. These differences, along with the convenience of taking pills as opposed to receiving an intravenous infusion, may make temozolomide plus PARP inhibition a more attractive option for some patients and providers.

\section{Potential biomarkers for temozolomide plus PARP inbibition}

Schlafen family member 11 (SLFN-11) regulates the response to DNA damage and replication stress; it can help halt the cell cycle in response to DNA damage (21). SLFN11 expression on tumor cells by immunohistochemistry was associated with improved survival for temozolomide plus veliparib, but expression was not associated with improved survival for temozolomide monotherapy. With this combination the median PFS was 5.7 months in patients with SLFN-11 expression $(n=12)$ versus 3.6 months in patients without expression $(\mathrm{n}=14), \mathrm{P}=0.009$. Similarly, the median OS was 12.2 versus 7.5 months for patients with and without SLFN-11 expression, $\mathrm{P}=0.014$ (8). In patient derived xenografts, SLFN-11 messenger RNA expression was also associated with improved outcomes with temozolomide plus olaparib (7).

There is currently no test of SLFN-11 expression that is approved for use in human samples and larger sample sizes are needed to validate this potential biomarker. Similarly, it remains to be determined whether expression of SLFN-11 on archival tissue will have similar utility to predict efficacy of temozolomide plus PARP inhibition when compared to levels of SLFN-11 on new biopsies/fresh tissue. Whether SLFN-11 RNA expression or SLFN-11 protein expression by immunohistochemistry are better at predicting patients who may derive longer term benefit from this combination requires further study.

Other biomarkers that have been investigated in human samples and which were not found to associate with efficacy of combination temozolomide plus PARP inhibition include PARP-1 expression by immunohistochemistry, O'6 methyl guanine DNA methyltransferase (MGMT) promotor methylation status and DNA repair defects in tumor tissue (8). However, it was hard to adequately evaluate the association of MGMT promoter methylation status and DNA repair defects with efficacy of combination temozolomide plus PARP inhibition because of small numbers of patients evaluable for these alterations (8).

\section{Retreatment with platinum-based doublets}

Retreatment with platinum-based doublets is considered in patients with platinum sensitive disease. Platinum sensitive disease has traditionally been defined as relapse $\geq 90$ days following receipt of most recent platinum-based regimen, while platinum resistant disease has generally been referred to as relapse $<90$ days following receipt of most recent platinum-based regimen. However, these definitions were developed in an era where there was no maintenance therapy following induction EP. Additionally, chemotherapy plus immune checkpoint inhibition is now considered the standard first-line therapy in many parts of the world for patients with ES-SCLC. Patients treated with this chemoimmunotherapy receive continuation maintenance with immune checkpoint inhibition. It is possible that this 90-day cut point may no longer help to optimally determine platinum sensitive versus resistant patients and that other biomarkers (e.g., inflammatory gene signatures or SLFN11 expression) could be investigated in this regard. The definition of platinum sensitive versus resistant disease needs to be revised in the current era of continuation maintenance with immune checkpoint inhibition following 
induction chemoimmunotherapy.

Never the less, some patients with relapsed ES-SCLC and a long time since receipt of their last platinum-based chemotherapy may be candidates for retreatment with platinum-based doublets. Retrospective studies have suggested potential efficacy of retreatment with platinumbased doublets for patients with platinum sensitive disease when using the old definition, which employed the 90-day time point to distinguish platinum sensitivity. In these retrospective studies the ORR ranged from $34 \%$ to $65 \%$. In one of these studies the median PFS was 5.5 months. The OS across these studies ranged from 6.3 to 14.4 months. However, randomized studies have not been conducted comparing retreatment with platinum-based doublets versus other subsequent line systemic therapy options in patients with platinum sensitive disease (21). Whether retreatment with platinum-based doublets is similarly efficacious, inferior or superior to these other options in platinum-sensitive patients remains to be determined. Additionally, data is not available to determine what the median relapse time was following receipt of platinum-based doublets for patients receiving retreatment on these retrospective studies. In essence, could the encouraging ORR and survival seen in these patients have been biased by highly platinum sensitive patients (e.g., those with relapse 6-12 months or more following receipt of last platinum-based therapy)? Similarly, did site of relapse (CNS versus extra-CNS) or number of progressing sites bias in any way the outcomes observed in these retrospective studies?

\section{Topotecan or amrubicin}

Topotecan is a topoisomerase I inhibitor (1-4). It is an approved second line chemotherapy for ES-SCLC. The ORR in unselected patients is $10-24 \%$, with ORR being greater in platinum sensitive versus platinum resistant patients. Platinum sensitive patients demonstrate an ORR of $17 \%$ to $27 \%$ and platinum resistant patients demonstrate an ORR of $5-13 \%$. The median PFS in these studies ranged from 2.8 to 3.8 months. The median OS ranged from 5.8 to 8.4 months, with OS being greater in platinum sensitive versus resistant patients. The main side effects observed with topotecan are cytopenias (1-4).

Amrubicin is a topoisomerase II inhibitor (4). It is an approved second-line chemotherapy option in some parts of the World for patients with ES-SCLC. It was compared to topotecan in a randomized phase III study. The investigator assessed ORR was numerically higher with amrubicin, 31\% versus $17 \%$; however, the median investigator assessed PFS and OS were not significantly different. Importantly, amrubicin was associated with more grade $\geq 3$ infections and a higher incidence of febrile neutropenia (4).

\section{Other chemotherapies}

The regimen of cyclophosphamide plus adriamycin plus vincristine (CAV) was compared to topotecan in a randomized study. CAV demonstrated similar efficacy as topotecan. However, symptom improvement was greater with topotecan (3).

Other chemotherapies have also been evaluated as second or subsequent line treatment in ES-SCLC: irinotecan, paclitaxel, gemcitabine, vinorelbine and others (21). While these therapies work for some patients, their outcomes are generally poor. The median PFS for these other chemotherapies ranges from 2 to 4 months and the median OS ranges from 4 to 7 months (21). These latter chemotherapies are sometimes used in patient management despite lacking formal approvals for ES-SCLC.

\section{Anlotinib}

Anlotinib is a multi-kinase inhibitor that most potently inhibits vascular endothelial growth factor receptor, KIT proto-oncogene receptor tyrosine kinase, platelet derived growth factor receptor and fibroblast growth factor receptor. This drug has been evaluated as third or later line treatment in patients with relapsed SCLC. On a phase II study patients were randomized to anlotinib or placebo. Anlotinib was administered orally at $12 \mathrm{mg}$ daily dosing on days $1-14$ of a 21 -day cycle. It is important to note that on this trial there were $40.7 \%$ of patients (33 of 81 ) on the anlotinib arm who were never smokers and $28.9 \%$ of patients ( 11 of 38 ) on the placebo arm who were never smokers. This high percentage of never smokers is uncharacteristic of a SCLC patient population and could have favorably biased outcomes. Approximately $63 \%$ of patients on this study were not platinum sensitive, with a relatively equal distribution between the two study arms. The percentage of patients having received prior immune checkpoint inhibition was not provided (22).

The ORR was negligible with anlotinib at about $5 \%$. However, the median PFS was significantly improved with anlotinib compared to placebo, median PFS of 4.1 versus 0.7 months, HR $0.19, \mathrm{P}<0.0001$. Similarly, the median OS was improved with anlotinib compared to placebo, with a 


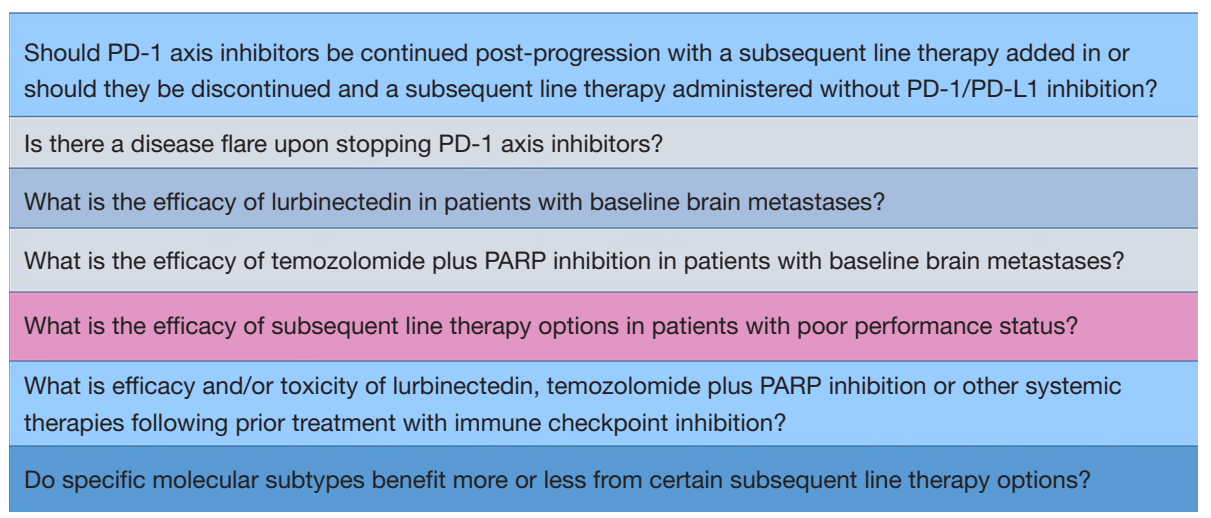

Figure 1 Unanswered questions in subsequent line therapy of ES-SCLC. ES-SCLC, extensive-stage small-cell lung cancer; PD-1, programmed death-1; PD-L1, programmed death ligand-1; PARP, poly ADP ribose polymerase.

median OS of 7.3 versus 4.9 months, HR $0.53, \mathrm{P}=0.0210$. This medication was relatively well tolerated, with the main adverse events consisting of hypertension (39.5\% all grades), hand-foot syndrome ( $21 \%$ all grades), diarrhea (16\% all grades), ALT elevation (16\% all grades) and proteinuria (12.3\% all grades) (Table 1) (22).

On the phase II trial of anlotinib discussed above patients with brain metastases could enroll if they had stable, previously treated brain metastases or asymptomatic untreated brain metastases. A subgroup analysis was conducted looking specifically at the patients with baseline brain metastases. Of the 21 patients with baseline brain metastases who received anlotinib there were $47.6 \%$ of patients who were never smokers, again not typical of a SCLC patient population. The percentage of those with baseline brain metastases on the placebo arm who were never smokers was $0 \%$. There were approximately $75 \%$ of patients on the anlotinib arm with baseline brain metastases who had previous radiation therapy to the CNS, while only $44 \%$ of patients with baseline brain metastases on the placebo arm had previous radiation to the CNS. The intracranial ORR was not reported for patients on either arm. The median PFS was significantly improved with anlotinib treatment in patients with baseline brain metastases when compared to placebo, median 3.8 versus 0.8 months, HR $0.15, \mathrm{P}=0.0005$. Similarly, the median OS was improved in patients with baseline brain metastases treated with anlotinib at median OS of 6.1 versus 2.6 months, HR $0.26, \mathrm{P}=0.0061$ (23). The similar PFS and OS hazard ratios in patients with baseline brain metastases when compared to the whole study population suggest that anlotinib may not have a CNS liability. However, further follow-up and potential evaluation in patients with untreated brain metastases is needed to more optimally determine the CNS efficacy of anlotinib.

\section{Other treatments and drugs in development}

Other drugs in development and their potential efficacy in ES-SCLC will be discussed in a separate section of this focused issue.

\section{Discussion}

Several second and subsequent line systemic therapy options have been discussed in this manuscript. An important question with all of these regimens is whether one should continue the PD-1 axis inhibitor and add in one of these systemic therapies or whether the PD-1 axis inhibitor should be discontinued and one of these systemic therapies initiated (Figure 1). It is unknown whether and to what extent a disease flare may be seen on discontinuation of PD-1 axis inhibitors. Additionally, some of the second and subsequent line therapy options may enhance the efficacy of immune checkpoint inhibition (e.g., lurbinectedin or temozolomide plus PARP inhibition). Such a question could be evaluated in the context of a cooperative group trial, with patients being randomized to continuation PD-1 axis inhibition plus a predefined subsequent line therapy option versus a predefined subsequent line therapy option without PD-1 axis inhibition.

Lurbinectedin monotherapy and temozolomide plus PARP inhibition are emerging as potential second line systemic therapy options following development of progressive disease on chemoimmunotherapy. The frequency of brain metastases is estimated at up to $80 \%$ during the disease course in 
patients with ES-SCLC (24). Unfortunately, neither of these combinations have reported intracranial response rates or intracranial PFS for patients with brain metastases (5-8). It is unknown whether lurbinectedin has CNS penetration. In contrast, temozolomide and PARP inhibitors penetrate the CNS. Additionally, temozolomide monotherapy has shown efficacy in SCLC brain metastases and primary brain tumors $(7,8,13,14)$. On the temozolomide plus veliparib study, about $20 \%$ of patients in each treatment arm (combo therapy and temozolomide monotherapy) had baseline brain metastases. These brain metastases had to be stable and asymptomatic, but they did not have to be previously treated (8). On the temozolomide plus olaparib study there were $40 \%$ of patients with baseline brain metastases. Patients with asymptomatic, untreated brain metastases $<1$ centimeter or previously treated and stable brain metastases were permitted to enroll on this latter study (7). Unfortunately, neither of these temozolomide plus PARP inhibitor studies reported data specific to patients with brain metastases or compared outcomes in patients with baseline brain metastases to those patients without baseline brain metastases $(7,8)$. Thus, it is not possible to determine the CNS efficacy of this combination or whether or not there is a CNS liability of this regimen (Figure 1). On the lurbinectedin monotherapy study only $4 \%$ of 105 patients had a history of CNS metastases $(6,11)$. On the lurbinectedin plus doxorubicin study only 1 of 27 patients (4\%) had a history of CNS metastases (5). The rate of CNS relapse with lurbinectedin monotherapy was $<10 \%$ on the phase II study and the rate of CNS relapse on the lurbinectedin plus doxorubicin study was 9 of 27 patients $(33 \%)(5,6,11)$. It is important to note that these CNS relapse rates with lurbinectedin monotherapy or combination were in patients with essentially no baseline CNS metastases. It is possible that using lurbinectedin monotherapy or combination in patients with baseline CNS metastases could be associated with significantly higher rates of CNS relapse than reported in studies to date and further evaluation of lurbinectedin in patients with a history of CNS metastases (both previously treated and untreated) is essential (Figure 1).

The majority of patients treated with lurbinectedin monotherapy (about 92\%) did not receive prior immune checkpoint inhibition $(6,11)$. Additionally, data on percentage of patients having received prior immune checkpoint inhibition was not available for temozolomide plus PARP inhibition or lurbinectedin plus doxorubicin $(5,7,8)$. The other systemic therapy trials discussed in this manuscript were conducted before immune checkpoint inhibition was approved in SCLC and/or in clinical trials for this disease, allowing one to conclude that very few if any patients received prior immune checkpoint inhibition on these other chemotherapy trials. This is an important observation as we have observed unique and/or greater toxicities for some agents when administered following prior immunotherapy (e.g., tyrosine kinase inhibitors given after immunotherapy in non-small cell lung cancer). It is possible that following immune checkpoint inhibition that treatment with the systemic regimens discussed in this manuscript could have a different side effect and/or efficacy profile than highlighted in this review (Figure 1).

Lurbinectedin monotherapy and combination therapy with doxorubicin have both demonstrated efficacy in relapsed ES-SCLC (Table 1) $(5,6,11)$. However, the toxicity of the combination with doxorubicin is much greater than with lurbinectedin monotherapy (Table 1) $(5,6,11)$. Additionally, the survival outcomes appear to be similar between lurbinectedin monotherapy and combination therapy with doxorubicin (Table 1) $(5,6,11)$. Thus, unless the survival outcomes on the ATLANTIS study are clearly superior to lurbinectedin monotherapy, the monotherapy regimen will be likely to be what moves forward into the treatment landscape of ES-SCLC.

The systemic therapy regimens discussed in this manuscript were evaluated predominately in patients with ECOG performance status 0 and 1 . However, many patients with ES-SCLC have an ECOG performance status of $\geq 2$. What the efficacy and toxicity of the regimens discussed in this review would be in patients with poor performance status remains to be determined (Figure 1).

Recently, SCLC investigators have proposed a classification schema of four molecular subtypes (25). The relationship that these molecular subtypes have in regards to the efficacy of the different systemic regimens discussed in this review will be important to investigate further (Figure 1). Such investigation may help with optimal selection of second and subsequent line systemic therapy options.

ES-SCLC almost always relapses following firstline treatment with EP plus a PD-1 axis inhibitor. Thus, effective subsequent line systemic therapy options are paramount. In this manuscript we have highlighted second or subsequent line systemic therapy options that are presently available in the clinic or soon to be available in the clinic. Future investigation of these regimens in special patient populations (e.g., patients with baseline CNS metastases or poor performance status), as well as evaluation 
of toxicity and efficacy following immune checkpoint inhibition are important (Figure 1). Unfortunately, survival with these different second or subsequent line systemic therapies is still not great. Thus, it is essential to further improve on subsequent line systemic therapy options for patients with ES-SCLC.

\section{Acknowledgments}

Funding: None.

\section{Footnote}

Provenance and Peer review: This article was commissioned by the editorial office, Fournal of Thoracic Disease for the series "Small Cell Lung Cancer". This article has undergone external peer review.

Conflicts of Interest: The author has completed the ICMJE uniform disclosure form (available at http://dx.doi. org/10.21037/jtd.2020.03.67). The series "Small Cell Lung Cancer" was commissioned by the editorial office without any funding or sponsorship. JMP served as the unpaid Guest Editor for the series and serves as the unpaid editorial board member of Fournal of Thoracic Disease from Oct 2019 to Sep 2021. Advisory board/consulting role for AstraZeneca, Gerson Lehrman Group, Hengrui Pharmaceuticals, Novartis and Pfizer. Honorarium from Genentech and Takeda. Research funding from Pfizer to institution.

Ethical Statement: The author is accountable for all aspects of the work in ensuring that questions related to the accuracy or integrity of any part of the work are appropriately investigated and resolved.

Open Access Statement: This is an Open Access article distributed in accordance with the Creative Commons Attribution-NonCommercial-NoDerivs 4.0 International License (CC BY-NC-ND 4.0), which permits the noncommercial replication and distribution of the article with the strict proviso that no changes or edits are made and the original work is properly cited (including links to both the formal publication through the relevant DOI and the license). See: https://creativecommons.org/licenses/by-nc-nd/4.0/.

\section{References}

1. Ardizzoni A, Hansen H, Dombernowsky P, et al.
Topotecan, a new active drug in the second-line treatment of small-cell lung cancer: a phase II study in patients with refractory and sensitive disease. The European Organization for Research and Treatment of Cancer Early Clinical Studies Group and New Drug Development Office, and the Lung Cancer Cooperative Group. J Clin Oncol 1997;15:2090-6.

2. Reck M, Vicente D, Ciuleanu T, et al. Randomized Phase 3 Study of Nivolumab Monotherapy Versus Chemotherapy in Relapsed Small Cell Lung Cancer: Results From CheckMate 331. European Society of Medical Oncology Immuno-oncology Congress 2018. Geneva, Switzerland. Dec 13-16, 2018. Abstract number 489.

3. von Pawel J, Schiller JH, Shepherd FA, et al. Topotecan versus cyclophosphamide, doxorubicin, and vincristine for the treatment of recurrent small-cell lung cancer. J Clin Oncol 1999;17:658-67.

4. von Pawel J, Jotte R, Spigel DR, et al. Randomized phase III trial of amrubicin versus topotecan as second-line treatment for patients with small-cell lung cancer. J Clin Oncol 2014;32:4012-9.

5. Forster M, Moreno V, Calvo E, et al. Overall Survival for Lurbinectedin plus Doxorubicin in Relapsed SCLC: Results from an Expansion Cohort of a Phase Ib Trial. IASLC World Conference on Lung Cancer Annual Meeting 2018. Toronto, Canada. September 23-26, 2018. Abstract number P1.12-20.

6. Paz-Ares LG, Trigo Perez JM, Besse B, et al. Efficacy and safety profile of lurbinectedin in second-line SCLC patients: Results from a phase II single-agent trial. J Clin Oncol 2019;37:8506.

7. Farago AF, Yeap BY, Stanzione M, et al. Combination Olaparib and Temozolomide in Relapsed Small-Cell Lung Cancer. Cancer Discov 2019;9:1372-87.

8. Pietanza MC, Waqar SN, Krug LM, et al. Randomized, Double-Blind, Phase II Study of Temozolomide in Combination With Either Veliparib or Placebo in Patients With Relapsed-Sensitive or Refractory Small-Cell Lung Cancer. J Clin Oncol 2018;36:2386-94.

9. Ponce S, Calvo E, De Miguel MJ, et al. Lurbinectedin (L) Combined with Paclitaxel (P) or Irinotecan (I) in relapsed SCLC: results from two phase Ib trials. IASLC World Conference on Lung Cancer 2019. IASLC World Conference on Lung Cancer 2019. Barcelona, Spain. September 7-10 2019. Abstract number 1588.

10. Petty W. Lurbinectedin. Santa Monica, California. IASLC Targeted Therapies in Lung Cancer 2020. Feb 19-22 2020. Oral Presentation. 
11. Trigo J, Subbiah V, Besse B, et al. Lurbinectedin as secondline treatment for patients with small-cell lung cancer: a single-arm, open-label, phase 2 basket trial. Lancet Oncol 2020;21:645-54.

12. Clinicaltrials.gov. Accessed 2/25/2020.

13. Stupp R, Mason WP, van den Bent MJ, et al. Radiotherapy plus concomitant and adjuvant temozolomide for glioblastoma. N Engl J Med 2005;352:987-96.

14. van den Bent MJ, Baumert B, Erridge SC, et al. Interim results from the CATNON trial (EORTC study 2605322054) of treatment with concurrent and adjuvant temozolomide for $1 \mathrm{p} / 19 \mathrm{q}$ non-co-deleted anaplastic glioma: a phase 3, randomised, open-label intergroup study. Lancet 2017;390:1645-53.

15. Pietanza MC, Kadota K, Huberman K, et al. Phase II trial of temozolomide in patients with relapsed sensitive or refractory small cell lung cancer, with assessment of methylguanine-DNA methyltransferase as a potential biomarker. Clin Cancer Res 2012;18:1138-45.

16. Zauderer MG, Drilon A, Kadota K, et al. Trial of a 5-day dosing regimen of temozolomide in patients with relapsed small cell lung cancers with assessment of methylguanineDNA methyltransferase. Lung Cancer 2014;86:237-40.

17. de Bono J, Ramanathan RK, Mina L, et al. Phase I, Dose-Escalation, Two-Part Trial of the PARP Inhibitor Talazoparib in Patients with Advanced Germline BRCA1/2 Mutations and Selected Sporadic Cancers. Cancer Discov 2017;7:620-9.

18. Murai J, Zhang Y, Morris J, et al. Rationale for poly(ADPribose) polymerase (PARP) inhibitors in combination

Cite this article as: Pacheco JM. Systemic therapy options following first-line chemoimmunotherapy in small-cell lung cancer. J Thorac Dis 2020;12(10):6264-6274. doi: 10.21037/ jtd.2020.03.67 therapy with camptothecins or temozolomide based on PARP trapping versus catalytic inhibition. J Pharmacol Exp Ther 2014;349:408-16.

19. Palma JP, Wang YC, Rodriguez LE, et al. ABT-888 confers broad in vivo activity in combination with temozolomide in diverse tumors. Clin Cancer Res 2009;15:7277-90.

20. Smith MA, Reynolds CP, Kang MH, et al. Synergistic activity of PARP inhibition by talazoparib (BMN 673) with temozolomide in pediatric cancer models in the pediatric preclinical testing program. Clin Cancer Res 2015;21:819-32.

21. Pacheco J, Bunn PA. Advancements in Small-cell Lung Cancer: The Changing Landscape Following IMpower-133. Clin Lung Cancer 2019;20:148-60.e2.

22. Cheng Y, Wang Q, Li K, et al. Anlotinib as third-line or further-line treatment in relapsed SCLC: a multicentre, randomized, double-blind phase 2 trial. IASLC World Conference on Lung Cancer 2018. Toronto, Canada. September 23-26, 2018. Abstract number OA13.03.

23. Cheng Y, Wang Q, Li K, et al. The impact of Anlotinib for relapsed SCLC with brain metastases: a subgroup analyses of ALTER 1202. IASLC World Conference on Lung Cancer 2019. Barcelona, Spain. September 7-10 2019. Abstract number P2.12-26.

24. Slotman B, Faivre-Finn C, Kramer G, et al. Prophylactic cranial irradiation in extensive small-cell lung cancer. $\mathrm{N}$ Engl J Med 2007;357:664-72.

25. Rudin CM, Poirier JT, Byers LA, et al. Molecular subtypes of small cell lung cancer: a synthesis of human and mouse model data. Nat Rev Cancer 2019;19:289-97. 